\title{
Optimal Clustering Configuration for Long Wireless Sensor Networks Lifetime
}

\author{
Ahmed Elkousy \\ Computer Science \& Eng. Dept., \\ Faculty of Electronic Eng., \\ Menoufia University, Menouf \\ 32952, Egypt \\ ahmed.elqusy@el- \\ eng.menofia.edu.eg
}

\author{
Salah S. E. Abdelrahman \\ Computer Science \& Eng. Dept., \\ Faculty of Electronic Eng., \\ Menoufia University, Menouf \\ 32952, Egypt \\ salahshaban3@yahoo.co.uk
}

\author{
Ayman El-Sayed \\ Computer Science \& Eng. Dept., \\ Faculty of Electronic Eng., \\ Menoufia University, Menouf \\ 32952, Egypt \\ ayman.elsayed@el- \\ eng.menofia.edu.eg
}

\begin{abstract}
Analyzing the Stable election protocol (SEP) in wireless sensor networks and aiming at the problem of network structure as, unbalanced nodes distribution per cluster, random deployment of advanced nodes. unreasonable cluster-heads selectivity and undefined time period in which the nodes to become a cluster head. So, an optimal energy leach protocol (OLEP) is proposed. In this algorithm, the network structure constructed by using Voronoi tessellation, thus the balanced nodes deployment. Based on SEP, the node's residual energy is added in cluster-heads election. The information is transmitted with mode of multi hops between network nodes. The simulation experiments showed that compared to the classical LEACH and SEP, this algorithm balances the energy consumption of the entire network and significantly prolongs network lifetime.
\end{abstract}

Keywords: Wireless Sensor Network; Clustering; Round Rotation Time; Hierarchical Network.

\section{Introduction}

Wireless Sensor Network (WSN) is a self-organizing network system formed by a large number of sensor nodes, such as data sensing, wireless communication and microcomputing, which are deployed in the monitoring range.

According to the topology in WSN, the routing protocol of wireless sensor network can be divided into planar routing protocol and hierarchical routing protocol[1][2]. The LEACH [3]protocol is a Low-Energy Adaptive Clustering Hierarchy protocol as a classical algorithm in clustered routing protocols. It is clustered by the signal strength received by the node, and the cluster head node is selected in a random loop to balance the energy consumption of nodes within the network. The SEP [4] (Stable election protocol) algorithm is a kind of classical heterogeneous network clustering routing algorithm, which is based on the LEACH protocol. The SEP algorithm is proposed by G.
Maragdakis as a classical heterogeneous network clustering routing algorithm. This agreement is developed based on the LEACH protocol, adding the initial energy isomorphism of this element, using the second energy heterogeneous network. According to the heterogeneous characteristics of the network is divided into normal nodes and advanced nodes, and set a different cluster election probability, so that the cluster election probability of advanced nodes is bigger more than the normal node. So that the advanced nodes and normal nodes close to the same time to death and extend the stability of the network cycle.

In this paper, the wireless communication energy consumption model in the SEP protocol is used. The energy consumption of the data transmission is mainly composed of the energy consumed by the wireless transceiver and the power amplifier. As shown in Figure 1.

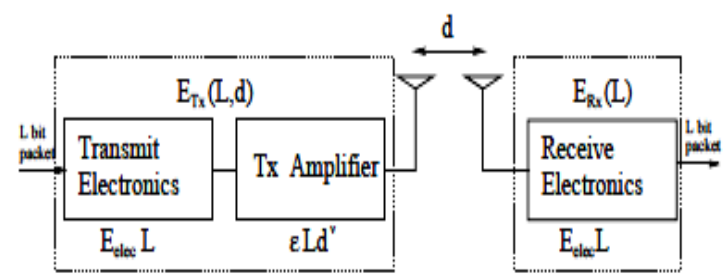

Figure 1: Energy Consumption Model.

The energy consumption of the power amplifier and the transmission environment are related to the transmission distance, and the energy consumption is divided into free space transfer mode and multipath transfer mode. The transmitter sends $L$ bits of energy consumed, as shown in Equation (1):

$$
\begin{aligned}
& E_{T x}(L, d) \\
& =\left\{\begin{array}{cc}
L . E_{\text {elec }}+L . \epsilon_{f s} \cdot d^{2} & \text { if } d \leq d_{0} \\
L . E_{\text {elec }}+L . \epsilon_{\text {amp }} \cdot d^{4} & \text { if } d>d_{0}
\end{array}\right\}
\end{aligned}
$$

In this equation: The $L$ represents the number of data bits; The $E_{\text {elec }}$ is the energy consumption of thetransceiver circuit: Thed is the distance between the sending node and the receiving node; The $d_{0}$ is the thresholdfor switching between free space and multipath mode and $d_{0}=\sqrt{\frac{\epsilon_{f s}}{\epsilon_{m p}}}$; 
The $\epsilon_{f s}$ and $\epsilon_{a m p}$ represent theenergy dissipation coefficients of the free-space and multipath models, respectively. Also, the radio transmitter of each member node is turned off until its transmission comes.Receiver energy consumption as shown in Equation (2):

$$
E_{T x}(L)=E_{R x}(L)=L . E_{\text {elec }}
$$

The SEP algorithm assigns each node a weight corresponding to the optimal probability $P_{\text {opt }}$, and its weight is the ratio of the initial energy of each node to the initial energy of the normal node. And define $P_{n r m}$ for the probability of normal node-weighted elections, $P_{a d v}$ for the probability of advanced node-weighted elections. Theformula is:

$$
\begin{gathered}
P_{n r m}=\frac{P_{o p t}}{(1+\alpha \cdot m)} \\
P_{a d v}=\left(\frac{P_{o p t}}{1+\alpha \cdot m}\right) *(1+\alpha)
\end{gathered}
$$

where $\alpha$ is the ratio of the initial energy of the higher node to the initial energy of the normal node. $m$ is theproportion of the advanced nodes in the total number of nodes. normal nodes and advanced nodes become cluster head threshold $T\left(S_{n r m}\right)$ and $T\left(S_{a d v}\right)$, as shown in Equation Error! Reference source not found. and Equation Error! Reference source not found.:

$$
\begin{aligned}
& = \begin{cases}\frac{P_{n r m}}{1-P_{n r m} \cdot\left(\operatorname{rmod} \frac{1}{P_{n r m}}\right)}, & S \in G^{\prime} \\
0 & \text { otherwise }\end{cases} \\
& = \begin{cases}\frac{T\left(S_{a d v}\right)}{1-P_{a d v} \cdot\left(r \bmod \frac{1}{P_{a d v}}\right)}, & S \in G^{\prime \prime} \\
0 & \text { otherwise }\end{cases}
\end{aligned}
$$

where $r$ is the current numbers of rounds.

The shortcomings of SEP algorithm are as follow:

1) The SEP algorithm is still a random selection cluster head mechanism using LEACH algorithm. The clustering process is to select whether to join the cluster according to the received cluster head node signal strength through the non-cluster head node. In this way, the cluster head is not evenly distributed, and its clustering method makes the number of nodes in the cluster random.

2) In the SEP algorithm, the election probability is only related to the initial energy of the node, but not with the residual energy: After the network is running for some time, the residual energy of the high-level node may not have the residual energy of the ordinary node, and the probability of becoming the cluster head is still higher than that of the ordinary node. This speed up the death of some advanced nodes and reduces the overall survival time of the network.

3) SEP algorithm is used in single-hop transmission mode, such a transmission so far away from the cluster head nodes need to spend a lot of energy to carry out longdistance information transmission, making the nodes in the network premature death, reducing the network life cycle.

4) The Sep algorithm does not determine the time for each node to become a cluster which is known as round rotation time, this period must be dedicated as during this period most power node is consumed.

Aiming at the above problems, an OLEP algorithm is proposed. The algorithm introduces the residual energy to optimize the cluster selection mechanism. The nodes in the network are organized into clusters by using the Voronoi tessellation, the multi hop transmission strategy is adopted in the cluster and determination of the optimal round rotation time for each node to become a cluster head to increase the network life time. Cluster head transports data to sink node through the multi-hop routing.

The rest of the paper is composed as follows. Section 0 contains a survey for the related works. Section 0gives the OELP protocol addressing the network model in Section 3.1, Protocol Overview in Section 3.2. In Section 3.3 we handle the optimal number of cluster head. After that Node deployment presented in Section 3.4. In Sections 3.5, and 3.6 we present the protocol Phases and the Optimal round rotation time. Section 0presents the performance evaluation of the proposed protocol. Section 0finishes up with the conclusion followed by the more relevant references.

\section{RELATED WORK}

In the literature[5], an improved stable voting protocol based on node energy consumption is proposed. The algorithm updates the next round of cluster election probability according to the consumption of each node in the round. The main purpose of improving the possibility which the node with higher residual energy is selected as cluster head is to balance the energy consumption of the node. In the literature[6], a distributed non-uniform clustering routing algorithm is proposed, which integrates the position factor and the average energy factor in the cluster selection mechanism to balance the residual energy of the whole network node. The main purpose of using the multi-hop dynamic routing between clusters is to balance the network energy consumption. In the literature[7], a clustering multi-hop routing protocol based on energy equalization is proposed. This algorithm can save the energy of nodes by adding relay nodes to assist in data forwarding and sharing the work of cluster head nodes in order to extend the entire network life cycle. In the literature[5][7], many optimization methods are proposed, but none of the nodes in the cluster are optimized. 


\section{OELP PROTOCOL}

\subsection{Network model}

In the study, in order to analyze the performance and energy consumption of OLEP, the following network model is assumed and shown in figure.

1) The location of the sink node is fixed and known outside of the monitoring area.

2) After the node is deployed, the location no longer changes.

3) Nodes have the same processing and communication capabilities and transmit power.

4) Node energy is heterogeneous way, divided into advanced nodes and normal nodes, the energy of advanced node is $(1+\alpha)$ times the energy of normal nodes, and all nodes can know their current residual energy.

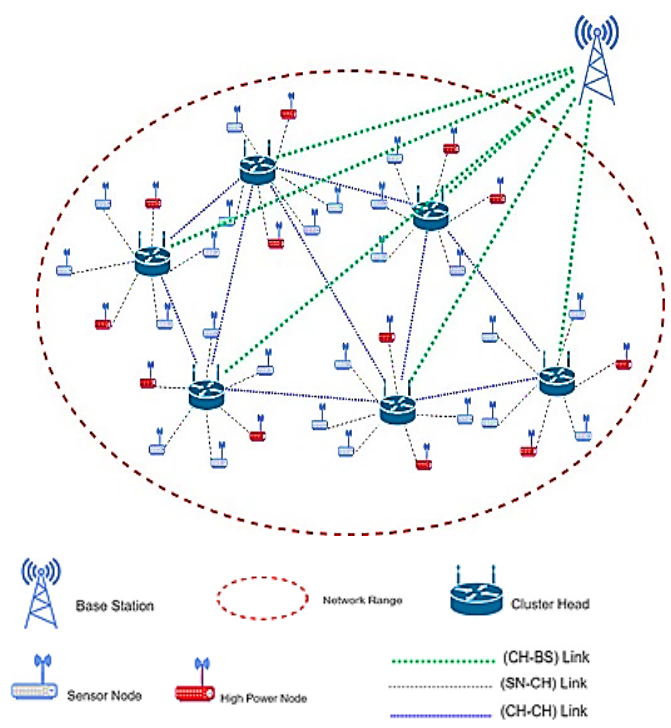

Figure 2: The Network Model with Advanced Node.

\subsection{Protocol overview}

Our model of a wireless sensor network with nodes inhomogeneous in their initial amount of energy. it presents the network model, the way to optimize the number of clusters, and the way to balance the distribution of the nodes inside the clusters.

Let us assume the case where a percentage of the population of sensor nodes is equipped with more energy resources than the rest of the nodes. Letmbe the fraction of the total number of nodes $n$, which are equipped with $\alpha$ times more energy than the others. We refer to these powerful nodes as advanced nodes, and the rest $(1-m) \cdot n$ as normal nodes.
In addition, every node transmits to the nearest $\mathrm{CH}$ in order to split the communication cost to the sink (which is many times greater than the processing and operation cost.) only the $\mathrm{CH}$ that report the sink and may consume a lot of energy, however this happens intermittently for every node. the round we define a time interval where all cluster members have to transmit to their cluster head in it more than message and we will determine this time later in this paper and the experiments will show the effect of this $R R T$

value. We show in this paper how the election process of cluster heads should be adapted appropriately to deal with heterogeneous nodes, which means that not all the nodes in the field have the same initial energy.

\subsection{Optimal Number of Clusters.}

The optimal construction of the network is basically depending on the determination of the number of the clusters. As this number must be dedicated and not a randomized selected value. $\operatorname{In}[3]$, the authors reached that if the clusters are not constructed in an optimal way, the total consumed energy of the sensor network per round is increased exponentially either when the number of clusters that are created is greater or especially when the number of the constructed clusters is less than the optimal number of clusters as shown in Figure 3[3]. From[3], the optimal number of clusters $K_{o p t}$ in the network is mathematically proved with Equation (5):

$$
K_{o p t}=\sqrt{\frac{N}{2 \pi}} \sqrt{\frac{\epsilon_{f s}}{\epsilon_{a m p}}} \frac{M}{d_{t o ~ B S}}
$$

For the experiments , $N=100$ nodes, $M=100 \mathrm{~m}$, $\epsilon_{f s}=10 \mathrm{pJ} \epsilon_{a m p}=0.0013 \mathrm{pJ} 75 \mathrm{~m}<d_{t o B S}<185 \mathrm{~m}$ so they said that the expect the optimum number of clusters to be $1<K_{\text {opt }}<6$ as shown in Figure 3.

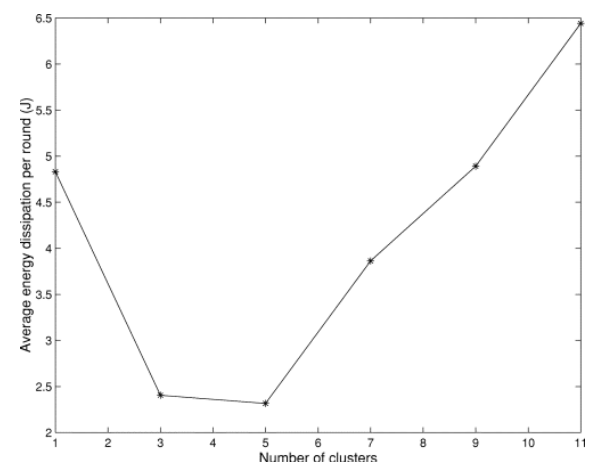

Figure 3: Average Energy Dissipation per Round as a Function of Number of Clusters.

Figure 3[3], which shows the average energy dissipated per round as a function of the number of clusters, shows that the simulation agrees well with the analysis. This graph shows that the optimum number of clusters is around 3-5 for the 100 -node network. When there is only one cluster, the noncluster head nodes often must transmit data very far to reach the cluster head node, draining their energy, and when there are more than five clusters, there is not as much local data 
aggregation being performed. For the rest of the experiments, they set to five.

\subsection{Node deployment}

In LEACH and SEP protocol, the clustering process is to select whether to join the cluster according to the received cluster head node signal strength through the non-cluster head node. In this way, the cluster head is not evenly distributed, and its clustering method makes the number of nodes in the cluster random. And in order to balance the energy dissipation, the clusters should contain approximately the same number of nodes. Recall that, after being selected to be the cluster heads, nodes broadcast an advertisement message to the rest of the nodes. The rest of nodes join the cluster based on the received advertisement's signal strength. The signal strength is determined by the distance between the nodes. The closer the distance, the stronger the signal is. Then the distance from the noncluster-nodes and corresponding cluster head is much closer than the other cluster heads. So, in order to ensure that the equalized deployment of the nodes inside the regions of the cluster. According to Voronoi tessellation deployment model, the target area is divided into $K_{\text {opt }}$ (optimal number of the calculated cluster which is equal-sized rectangular areas). By using the Voronoi tessellation, the distance for any non-clustered head to the corresponding cluster head is obtained.

In the well-divided area, the advanced nodes are equally deployed near the geometric center of the area, and the common nodes are deployed in the Voronoi calculated regions. As shown inFigure 4:

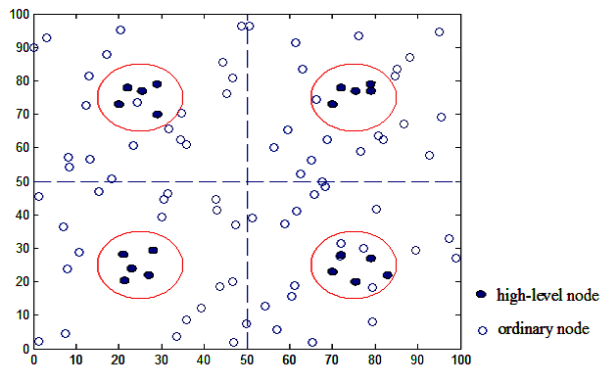

Figure 4: Node Deployment.

Given an network domain $\mathrm{M} * \mathrm{M}$, and the determined optimal number of cluster head $K_{o p t}$, by applying the rules of tessellation into our network parameters we will find that the network area can be divided into $\frac{M^{2}}{K_{o p t}}$ regions. Suppose the position of each cluster head $\left(x^{*}, y^{*}\right)$ is the center of its corresponding Voronoi region $\mathrm{Vi}$ and any non-cluster node position is $(\mathrm{x}, \mathrm{y})$. Since the nodes are uniformly distributed in the area of clusters. So, the expected distance from noncluster head node to the corresponding cluster head will beas follow in Equation (6)

$$
d_{\text {toCH }}^{2}=\frac{M^{2}}{3 K_{\text {opt }}}
$$

The maximum expected distance from any node to the corresponding cluster head in order to insure it will belong to it. So, it is cleared how to distribute the nodes in a network domain to ensure the equalized distribution of the nodes inside the clusters.

\subsection{OLEP Phases}

We consider a sensor network that is hierarchically clustered. The LEACH protocol [3] maintains such clustering hierarchy. The operation of OLEP based on the LEACH protocol, which is controlled through rounds, and consist of two phases: Setup Phase, and Steady State Phase.

1) Setup Phase:

Clusters are formed and cluster head $(\mathrm{CH})$ is elected for each cluster. In steady phase, data is sensed, aggregate, compressed and transmit to base station.

\section{Cluster head selection algorithm:}

In OLEP, the clusters are re-established in each "round". New cluster heads are elected in each round and as a result the load is well distributed and balanced among the nodes of the network. Moreover, each node transmits to the closest cluster head to split the communication cost to the sink (which is tens of times greater than the processing and operation cost.) Only the cluster head has to report to the sink and may expend a large amount of energy, but this happens periodically for each node. In OLEP there is an optimal percentage $p_{\text {opt }}=\frac{N}{K_{\text {opt }}}$ of nodes that has to become cluster heads in each round assuming uniform distribution of nodes in space[8], [9], [10], and [11].

Every node decides whether or not to become a cluster-head for the current round. This decision depends on the recommended rate of cluster heads set out toward the network (decided from the earlier) and Nodes that are elected to be cluster heads in the current round can no longer become cluster heads until all the nodes in the cluster becomes a cluster head for one round . but for the nodes that have power resources equal to $\alpha$ times than the normal nodes. it must become a cluster head $\alpha$ times than the normal nodes. So, the election probability for the advanced nodes differ from that of the normal nodes [4] and [5].

So, for the normal nodes its election probability is determined in Equation (3), and for the advanced nodes it will be Equation (4).

The decision is made at the beginning of each round by each node in $S_{n r m} \in G^{\prime}$ and $S_{a d v} \in G^{\prime \prime}$ independently choosing a random number in $[0,1]$. The Election of cluster heads are depending on threshold value $T(S)$. If the random number is less than a threshold $T(S)$ then the node becomes a cluster head in the current round. In this paper, the OLEP algorithm introduces the residual energy of the node in the cluster selection mechanism as shown in Equation (7) 


$$
\begin{aligned}
& T\left(\boldsymbol{S}_{n r m}\right) \\
& = \begin{cases}\frac{P_{n r m} * \boldsymbol{H}}{1-P_{n r m} \cdot\left(\operatorname{rmod} \frac{1}{P_{n r m}}\right)}, & S \in G^{\prime} \\
0 & \text { otherwise }\end{cases}
\end{aligned}
$$

Where $T\left(s_{n r m}\right)$ the threshold for normal nodes, and $r$ is the current round, $G^{\prime}$ is the set of normal nodes that have not become cluster heads within the current session (which the time period where all nodes in the cluster become a cluster head for one round ), and $T\left(s_{n r m}\right)$ is the threshold applied to a population of $n \cdot(1-m)$ (normal) nodes. This guarantees that each normal node will become a cluster head exactly once every session.Similarly, the threshold for advanced nodes, in Equation(8):

$$
= \begin{cases}\frac{T\left(\boldsymbol{S}_{a d v}\right)}{P_{a d v} * \boldsymbol{H}} & \\ 1-P_{a d v} \cdot\left(r \bmod \frac{1}{P_{a d v}}\right) & \\ 0 & \text { otherwise }\end{cases}
$$

where $G^{\prime \prime}$ is the set of advanced nodes that have not become cluster heads within the last session, and $T\left(\boldsymbol{S}_{a d v}\right)$ is the threshold applied to a population of $n \cdot m$ (advanced) nodes. This guarantees that each advanced node will become a cluster head exactly once every $1+\alpha$ time.

The previous equations applied in the real situation where the election probability is not only related to the initial energy of the node, but for the residual energy. In the SEP protocol the threshold equations for the normal and advanced nodes assume the ideal situation that the cluster head nodes consume the same amount of power and this Inconsistent with reality. In the real situation, After the network is running for some time, the residual energy of the advanced node may not have the residual energy of the normal node, but still the probability of becoming the cluster head is still higher than that of the normal node. This speed up the death of some advanced nodes and reduces the overall survival time of the network. So, the residual energy must become a factor in the cluster head selection equation. In this paper, the OLEP algorithm introduces the residual energy of the node in the cluster selection mechanism. In Equation(8), $H$ is the adjustment function:

$$
H(r)=\frac{E_{i}(r)}{E_{\max }(r)}
$$

where $E_{i}(r)$ is the residual energy of the node $i$ in the $r$ rounds; $E_{\max }(r)$ is the maximum value of residual energy in the $r$ rounds.

From(9)(9), we can see that the probability of the node being elected as cluster head increases when the node's residual energy increases, the threshold becomes larger.

\section{The cluster creation}

Every node that has chosen as a cluster head for the current round communicates and sends advertisement message to the rest of the nodes for formation of cluster. This is also called cluster-head advertisement phase. If non-cluster-head node receives high signal strength advertisement message then it needs minimum energy for data transmission with that cluster head so, non-cluster-head nodes make decision to form cluster on the basis of signal strength of advertisement message of cluster head. Then cluster head generates timeslot for all nodes. Only in that time slot nodes can transmit a data to cluster head. Schedule Creation is based on total number of nodes present in network. TDMA (Time Division Multiple Access) determines when node transmits the data to cluster head[12][13].

\section{2) Steady State Phase.}

During steady state stage, the sensor nodes i.e. the noncluster head nodes begins sensing information and send it to their cluster head as per their TDMA plan. The cluster head gather information from every one of the nodes in cluster and compress the information at that point then send it to base station or sink.

\subsection{Determination of Optimal Round Rotation Time (ORRT).}

Although a lot of work focus on optimizing LEACH protocol and prolong the network lifetime but they all neglected how much time the node must still as a cluster head which known as the round rotation time (RRT).This Period is very important factor (as this period in which the $\mathrm{CH}$ consumes most of its power). so we introduce a solution how to determine the optimal period for each node to become a cluster head whether this node is an original or high power node, and show how this period will effect on the network life time and its stable region which increase the network reliability that is important for a lot of application.

We derived this concept in a mathematical wayand verified it in an experimental way. For the period, if each round lasts too long, the cluster heads stay active all time, the energy of the cluster head may drain quickly. This will lead to early dead of the first node which decrease the stable period (is the period before the death of the first node in the network). this stable period is very important to become longer as we can because it is the most reliable period in the network lifetime, as the reliability of the collected data about the sensing environment is highest). On the other hand, when $R R T$ is too short, the network rebuilds the clusters frequently and then most of the energy will be wasted in the set-up phase instead of transmitting the data in the steady phase.

We focus on how long each round should last before the network restart another round with a new elected cluster head. By using Voronoi region to describe the distribution form of the cluster heads and their cluster members to obtain the optimal ORRT. Because of the restricted energy, the length of the steady stage ought to be longer than that of the set-up stage to achieve the information transmission. What 
is more, the set-up stage will cost a trivial power dissipation. For our selected simulated environment there are $\mathrm{N}$ nodes uniformly distributed in an $\mathrm{M} \times \mathrm{M}$ field.

Because of the base station $(B S)$ is as always far from the field, we consider separation to the BS is greater than the crossover distance, so power dissipation pursues the multipath model[14]. The power of each $\mathrm{CH}$ is utilized to get the information from its cluster individuals, aggregate the received information and send the collected information to BS. Thus, the energy dissipated in the $\mathrm{CH}$ nodes $i(i=1$, $2, \ldots n)$ during a single frame in the is as below:

$$
\begin{gathered}
E_{\text {ch }}=l E_{\text {elec }}\left(\frac{n}{K}-1\right)+l E_{D A} \frac{n}{K} \\
+E_{\text {elec }}+l \varepsilon_{\text {amp }} d_{i}^{4} \\
=l E_{\text {elec }} \frac{n}{K}+l E_{D A} \frac{n}{K}+l \varepsilon_{\text {amp }} d_{i}^{4}
\end{gathered}
$$

where $l$ is the number of bits in each message, Eelec is the power dissipated per bit, EDA is the power for collecting the information, $d i$ represents the separation (distance) between the $\mathrm{CH}$ node and the $\mathrm{BS}$. The non $-\mathrm{CHs}$ nodes are just to transmit information to its relating $\mathrm{CH}$. In the normal situation, we consider the separation from non $-\mathrm{CH}$ nodes for their relating $\mathrm{CH}$ is generally little, at that point the power dissemination pursues the Friss free-space model[14]. So, the power consumption of each non $-\mathrm{CH}$ is characterized as follow:

$$
\begin{gathered}
E_{\text {non }-c h}=l E_{\text {elec }}+l \varepsilon_{f s} d_{\text {toCH }}^{2} \\
\operatorname{EXP}\left(E_{\text {non }-c h}\right)=l E_{\text {elec }}+l \varepsilon_{f s} \operatorname{EXP}\left(d_{\text {toCH }}^{2}\right)
\end{gathered}
$$

where $\operatorname{EXP}\left(d_{t o C H}^{2}\right)$ is the Expectation distance of the member node to its cluster head. To calculate Equation (12), we should know the estimation of $d_{t o C H}$. The non-cluster head nodes join the cluster dependent on the gotten ADV's signal quality. The signal quality is dictated by the separation between the sensor nodes. The closer the distance, the stronger the advertise message (ADV) signal for the corresponding cluster head to the non-cluster head nodes is. At that point, the separation from the non- $\mathrm{CH}$ nodes and relating cluster head is a lot nearer than the other cluster heads. Furthermore, this is essentially how the Voronoi tessellation refine its area.

From Equation (6), Equation (11), and Equation (12), we obtain:

$$
E_{\text {non }-c h}=l E_{\text {elec }}+l \varepsilon_{f s} \frac{M^{2}}{3 K_{\text {opt }}}
$$

The total energy consumed by each node per round depends on the average number of frames per round can be write as:

$$
E_{C H \mid \text { round }}=N_{\text {frame } \text { |round }} x E_{C H \mid \text { frame }}
$$

$$
E_{\text {non }-C H \mid \text { round }}=N_{\text {frame } \mid \text { round }} x E_{\text {non-CH } \mid \text { frame }}
$$

where $E_{C H \mid \text { round }}$ is the power which $\mathrm{CH}$ goes to get information from their cluster individuals, collect and process information and speak with the base station, $N_{\text {frame |round }}$ is the normal number (average) of frames per round.

$E_{\text {non-CH|round }}$ is the power utilized by non-cluster head in order to send information to their comparing $\mathrm{CH}$. The base time (minimum) for each round ought to be sufficiently adequate for enabling a sensor node to be $\mathrm{CH}$ in any event once and non- $\mathrm{CH}$ numerous times, before the power is spent. For instance, if there are $\frac{n}{K_{o p t}}$ rounds in the sensor network activity, we should ensure that the power of every sensor node can continue assuming the job of $\mathrm{CH}$ once and cluster member $\left(\frac{n}{K_{o p t}}-1\right)$ times. According to Equation (10) Equation (13),Equation (14) and Equation (15), we get:

$$
\begin{gathered}
E_{\text {total }}=E_{\text {CH|round }}+\left(\frac{n}{K_{\text {opt }}}-1\right) E_{\text {non }-C H \mid \text { round }} \\
=N_{\text {frames |round }}\left(l E_{\text {elec }} \frac{n}{K_{\text {opt }}}+l E_{D A} \frac{n}{K_{\text {opt }}}\right. \\
\left.+l \varepsilon_{\text {amp }} d_{i}^{4}\right)+ \\
N_{\text {frames |round }}\left(\frac{n}{K_{\text {opt }}}-1\right)\left(l E_{\text {elec }}+l \varepsilon_{f s} \frac{M^{3}}{3 K_{\text {opt }}}\right) \\
=\frac{E_{\text {total }- \text { start }}}{0.0068}
\end{gathered}
$$

In WSN, the bit rate is a predefined as a consistent value $R_{b}$ , the time of transmitting one bit is then indicated as $t_{b i t}=\frac{l}{R_{b}}$.As there are $\frac{n}{K_{o p t}}$ nodes in the cluster, and the complete time for all sensor nodes in the cluster to transmit one message is:

$$
t_{\text {frame }}=\frac{n}{K_{\text {opt }}} \frac{L}{R_{b}}
$$

As the total number of nodes is $100+(20 \% * 3)=160$ nodes

$$
t_{\text {frame }}=\frac{160}{10} * \frac{4000}{10^{6}}=0.064 \mathrm{sec}
$$

From Equations (16) and Equation (17) The time of each round is then

$$
t_{\text {round }}=N_{\text {frames } \mid \text { round }} X t_{\text {frame }}
$$$$
=\frac{n}{K_{\text {opt }} R_{b}} \frac{E_{\text {total }- \text { start }}}{\left.\left[\left(E_{\text {elec }} \frac{n}{K_{o p t}}+E_{D A} \frac{n}{K_{o p t}}+\varepsilon_{\text {amp }} d_{i}^{4}\right)\right]\left(\left(\frac{n}{K_{o p t}}-1\right)\left(E_{\text {elec }}+\varepsilon_{f s} \frac{M^{3}}{3 K_{\text {opt }}}\right)\right)\right]}
$$ 
To make the network more energy-efficient, we should consider all the clusters and prolong the time for one round as long as possible. So, from Equation Error! Reference source not found. the optimal round rotate time should be.

$$
\begin{aligned}
& t_{\text {ORRT }}= \\
& \begin{aligned}
\max \left(\frac{n}{K_{\text {opt }} R_{b}} \frac{E_{\text {total }- \text { start }}}{\left[\left(E_{\text {elec }} \frac{n}{K_{\text {opt }}}+E_{D A} \frac{n}{K_{\text {opt }}}+\varepsilon_{\text {amp }} d_{i}^{4}\right)\right]\left(\left(\frac{n}{K_{\text {opt }}}-1\right)\left(E_{\text {elec }}+\varepsilon_{f s} \frac{M^{3}}{3 K_{\text {opt }}}\right)\right]}\right) \\
\quad=0.064 * \frac{E_{\text {total }- \text { start }}}{.0068}
\end{aligned}
\end{aligned}
$$

For our assumption, the $E_{\text {total-start }}=0.5 \mathrm{j}$. So, by applying in Equation Error! Reference source not found. we get

$$
t_{\text {ORRT }} \cong 5 \mathrm{sec}
$$

\section{Performance Evaluation}

\subsection{Simulation Setup}

Our simulation is a wireless sensor network in a clustering structure in a domain with dimensions $100 \mathrm{Mx} 100 \mathrm{M}$. The aggregate number of sensor nodes $\mathrm{n}=100$. The sensor nodes, both original and high-power nodes, are arbitrarily (uniformly) conveyed over the field. This uniformity implies that the horizontal and vertical coordinates of every sensor node haphazardly chosen among 0 and the most extreme estimation of the dimensions. The sink is far away in a safe location. The initial power of a typical sensor node-set to $\mathrm{E}_{\text {total }- \text { start }}=0.5$ Joules, Although this value is randomly for the task of this study, this does not influence the behavior of our OELP protocol. The radio features utilized in our experiment appear in Table 1. The message length that nodes send to their $\mathrm{CH}$ and the length of the (total)

\begin{tabular}{|c|c|c|}
\hline Description & Parameter & Value \\
\hline $\begin{array}{l}\text { Radio electronic } \\
\text { energy }\end{array}$ & $\mathrm{E}_{\text {elec }}$ & $50 \mathrm{~nJ} / \mathrm{bit}$ \\
\hline $\begin{array}{c}\text { Compute energy } \\
\text { for data } \\
\text { aggregation }\end{array}$ & $\mathrm{E}_{\mathrm{DA}}$ & $5 \mathrm{~nJ} / \mathrm{bit}$ \\
\hline $\begin{array}{l}\text { Base station } \\
\text { location }\end{array}$ & $\mathrm{L}_{\mathrm{BS}}$ & $(150,50)$ \\
\hline Number of nodes & $\mathrm{n}$ & $50,100,200$ \\
\hline Network Size & M X M & $100 \times 100$ \\
\hline Data size & $\mathrm{L}$ & 4000 bits \\
\hline Bitrate & $\mathrm{R}_{\mathrm{b}}$ & $1 \mathrm{M} \mathrm{bps}$ \\
\hline Initial energy & $\mathrm{E}_{\text {total -start }}$ & $0.5 \mathrm{~J}$ \\
\hline $\begin{array}{l}\text { Transmit } \\
\text { Amplifier } \\
\text { if } \mathrm{d}_{\mathrm{toBS}} \geq \mathrm{d} 0\end{array}$ & $\epsilon_{\mathrm{fs}}=$ & $\frac{\frac{10 \mathrm{pJ}}{\mathrm{bit}}}{\mathrm{m}^{2}}$ \\
\hline
\end{tabular}
message that a group goes to the sink set to 4000 bits.

Table 1: Notations of Radio Features for Sensor Node in our Experiment.

$$
\begin{aligned}
& \text { Transmit } \quad \epsilon_{\mathrm{mp}}=\quad \frac{\frac{0.0013 \mathrm{pJ}}{\mathrm{bit}}}{\mathrm{m}^{2}} \\
& \text { Amplifier } \mathrm{d}_{\mathrm{toBS}}>\mathrm{d} 0
\end{aligned}
$$

In the following subsections, we will simulate the OELP protocol, within sight of heterogeneity in the initial power of the sensor nodes. We assess the behavior of the two protocols to the performance measures that mentioned before. We additionally look at the sensitivity of our protocol to the level of heterogeneity in the sensor network - which means the length of the stability region by varying $\mathrm{m} \& \alpha$. We first outline our general observations:

- Our OLEP protocol successfully extends the stable region by being aware of centralizing of the high-power nodes and through assigning probabilities of cluster-head election weighted by the relative residual energy of nodes.

- Due to extended stability, the throughput of OLEP is also higher than that of current heterogeneous clustering protocols.

- The performance of OLEP is observed to be close to that of an ideal upper bound obtained by distributing the nodes uniformly over in the sensor field using the Voronoi tessellation.

- OLEP is more resilient than SEP and LEACH in judiciously consuming the extra energy of advanced nodes.

\subsection{Evaluation Metrics}

We characterize here the measures we use in this research paper to measure the clustering protocols performance.

Stability Period: is the time interval from the start of network operation until the death of the first sensor node.

We also refer to this period as "stable region."

- Instability Period: is the time interval from the death of the first node until the death of the last sensor node.

We also refer to this period as "unstable region."

- Network lifetime: is the time interval from the start of operation (of the sensor network) until the death of the last alive node.

- Number of cluster heads per round: This instantaneous measure reflects the number of nodes which would send directly to the sink information aggregated from their cluster members.

- Number of alive (total, advanced and normal) nodes per round: This instantaneous measure reflects the total number of nodes and that of each type that have not yet expended all their energy.

- Throughput: We measure the total rate of data sent over the network; the rate of data sent from cluster heads to the sink as well as the rate of data sent from the nodes to their cluster heads. 
The bigger the stability region and the littler the Unstable region is, the better the dependability of the clustering procedure of the sensor network. Then again, there is a tradeoff between dependability and the lifetime of the network system. Until the point that the death of the last node we can, in any case, have a few criticisms about the sensor field even though this input may not be dependable. The lack of reliability of the feedback originates from the way that there is no assurance that there is at least one cluster head per round amid the last rounds of the task. In our model, the nonappearance of a $\mathrm{CH}$ in an area prevents detailing the region to the sink node. The throughput measure captures the rate of such information reporting to the base station.

\subsection{Results}

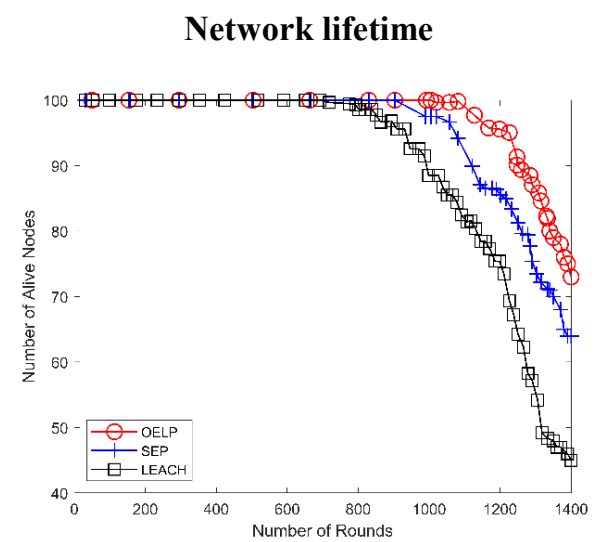

Figure 5: Network Lifetime as number of Alive Nodes per round

Figure 5 shows the number of surviving nodes per round. The number of OLEP algorithm survives the other two algorithms. And the death time of the first node in the network is significantly longer than that of the other two algorithms. This is due to OLEP algorithm using a Voronoi tessellation algorithm, so that close nodes are concentrated in the same cluster. This not only reduces the node consuming power as it reduces the average path length of the cluster nodes to the cluster head, but also make all cluster contain approximately the same number of the nodes.

In the OLEP algorithm, the information transmission in the cluster is done by multi-hop. The nodes which in the cluster cannot communicate directly with the cluster head forward the information to the cluster head through the other nodes in the cluster. The multi-hop transmission reduces the energy consumption required by a single node for longdistance transmission to the cluster head. And the information transmission between cluster heads still adopts multi-hop routing strategy. The cluster head node, which is far from the sink node, transmits the information to the cluster head near the convergence node. Through the forwarding between the cluster heads is to reduce the longdistance transmission of energy consumption. Thus, the energy of a single node which need carry out long distance transmission is distributed to the forwarding node. Thus, not only balance the network energy consumption, but also extend the network survival time.

\section{Remaining Energy}

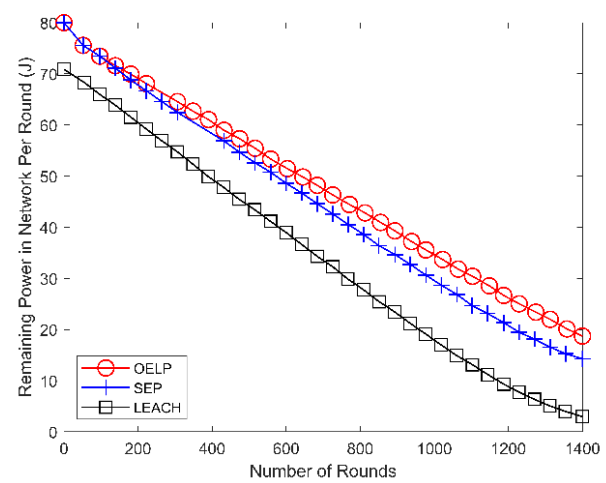

Figure 6: Remaining Energy in the Network per Round

Figure 6 shows the total energy remaining for each round of the network. The total remaining energy of the OLEP algorithm is more than the other two algorithms in the network. When the advanced node is deployed in the target area, the advanced nodes deployed in the geometric center of the cluster are closer to the rest of the nodes and are easier to communicate with the rest of the nodes, as these nodes have probability to be a cluster head higher than the normal nodes; On the contrary, the normal nodes are at the edge of the cluster domain. According to the cluster selection mechanism of OLEP algorithm, nodes with high power are more likely to serve as cluster heads. And nodes with centralized positions have the probability of forwarding tasks, so the geometric center of the cluster belongs to the concentrated area with high energy consumption. The multihop transmission is done by deploying the advanced node in the geometric center of the cluster. So, the OLEP algorithm can balance the network energy consumption and extend the network life cycle.

\section{Throughput Rate}

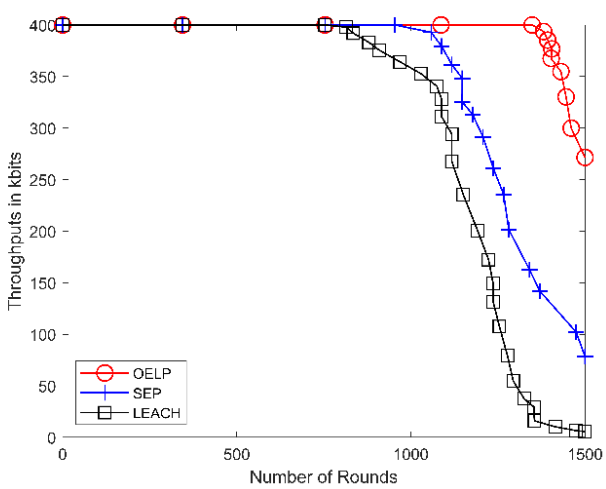

Figure 7: Throughput of the Network Per Round 
Figure 7 demonstrates the throughput from $\mathrm{CHs}$ to the base station. The throughput of OELP is fundamentally bigger than that of LEACH and SEP in the stable period, and for the majority of the unstable period. This implies in light of the fact that OELP ensures CHs in more rounds then these $\mathrm{CHs}$ will report to the sink due to the longer network lifetime. In addition, similar results are seen in Figure 13 for the throughput of sensor nodes to their $\mathrm{CHs}$, as the $\mathrm{CHs}$ in the case of OELP are chosen in a way that more stable form during the unstable period. Therefore, the overall throughput of OELP is greater than that of LEACH and SEP.

\section{Round rotation time}

In the experiments, we observed the network system lifetime for different values of RRT in the network and show experimentally the RRT value we determined effectively increase the network lifetime. Likewise, we plotted the changes of power utilization and the quantity of alive sensor nodes over time explicitly for a 100 sensor nodes network with various RRT settings. The results of the experiments outlined the RRT we conducted is significantly more power- effective also, keep more nodes alive.

With the settings in the notation table, we acquire the ideal ORRT $=5$ secs in every one of the three unique systems. In wireless sensor network there are different understanding for the network's death. Here, we adopted a popular one to consider the network system is down when the quantity of alive sensor nodes is less than the number of the clusters.

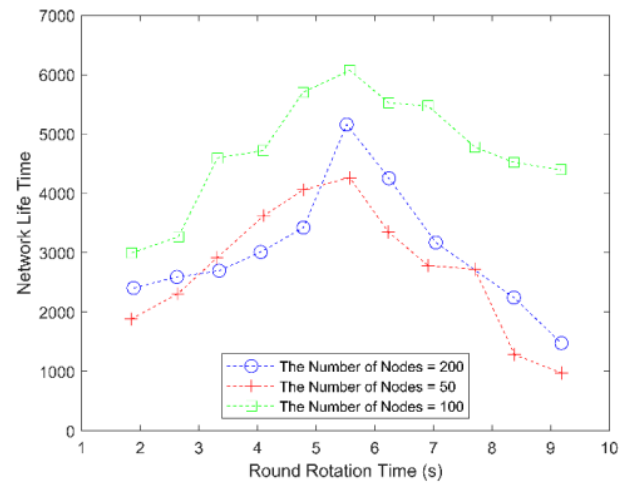

Figure 8: the network lifetime achieves the peak when RRT $=5$ in three different situations,

Figure 8demonstrates the network lifetime achieves the peak when $\mathrm{RRT}=5$ in three different situations, which is predictable with our calculated optimal value. We can likewise observe that the lifetime of system with 100 sensor nodes is any longer than other two cases. We can observe that the lifetime of system with 200 sensors is longer than 50 sensor nodes. That is on the basis that that the density of the sensor nodes in the network domains is also another factor to influence the lifetime of system for LEACH which anyway won't be talked about further in this paper.

While Figure 9demonstrates the number of alive sensor nodes with various RRT setting in the 100 -sensor nodes network. We can see that, with the RRT setting as $\approx 5$ seconds, the alive number is decreased all the more gradually after some time than that of others. Also, clearly the primary dead sensor node comes up later than the others. When we set the RRT excessively long, say 7 seconds, we can see that the primary dead sensor node comes up a much prior and the lifetime is shorter than others. This is on the basis that in steady stage $\mathrm{CHs}$ keep alive constantly and power get disseminated soon. In the case that we set the RRT.

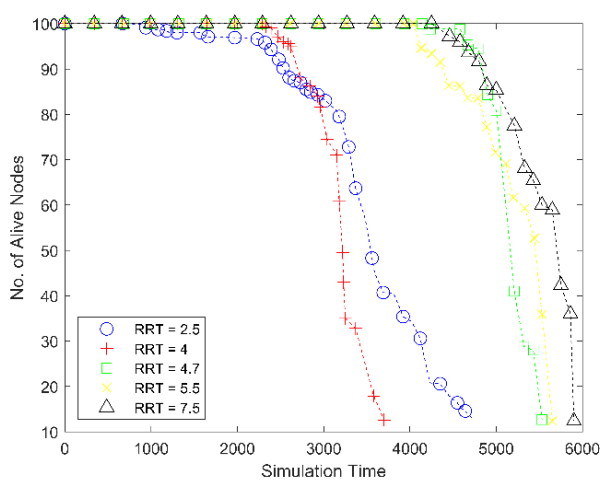

Figure 9: Number of alive node per Round with Difference RRT

Excessively short, say 2 seconds, the power gets depleted likewise soon. This is on the basis that the system changes $\mathrm{CHs}$ too frequently and as a result that the power dispersed in the setup stage isn't insignificant any more as the number of rounds expands a lot.

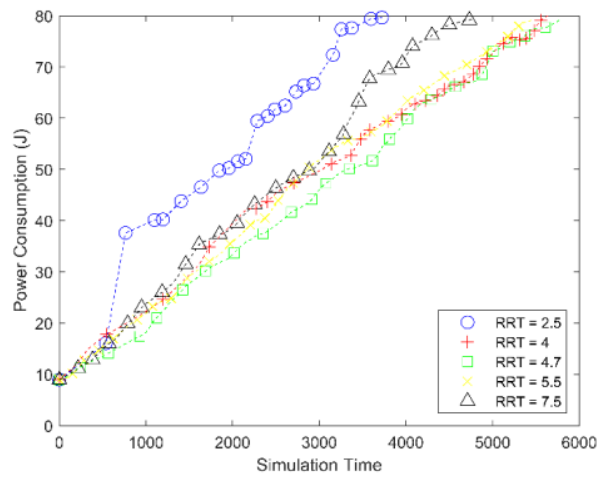

Figure 10: Power Consumption per round with different RRT.

We also made a comparison in the power utilization with different RRT in the 100 -sensor nodes network. FromFigure 10 , we can see that the RRT we got (the RRT $\approx 5$ ) consume least energy at most of the time. Once more, on one hand we can see when the RRT is excessively long the power diminishes rapidly. Then again, if that it is excessively short, the power decreases most rapidly, as the proportion set-up stage in one round increased, and now set-up stage require more power than steady stage every second.

From Figure 11, we see that the information received at BS is the most when the RRT is around 5 seconds. In the case that the RRT is excessively long, the sensor nodes deplete its power quickly, at that point the information creates at $\mathrm{CH}$ 
decrease which lead to the information received at BS diminish. In the event that the RRT is excessively short, the time specified for steady stage diminishes and more time is squandered in set-up stage which is useless for information transmission. So, the information received at BS sharply diminishes.

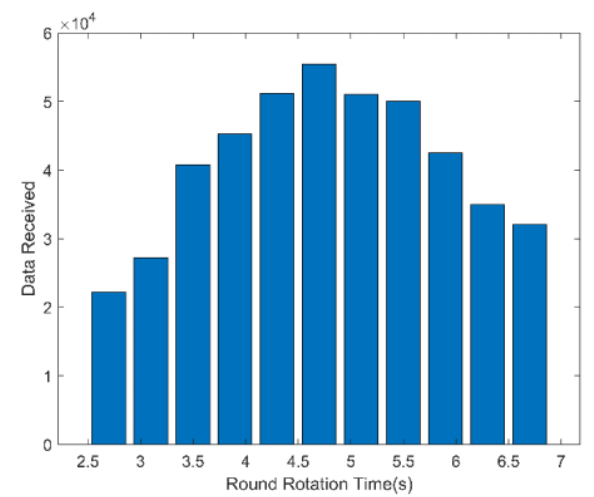

Figure 11:Date Received in the network With Different RRT

\section{CONCLUSIONS AND FUtURE WORK}

We proposed OELP (Optimal Energy LEACH Protocol) so every sensor node in a heterogeneous two-level various hierarchal network freely chooses itself as a $\mathrm{CH}$ dependent on its initial power with respect to that of other sensor nodes. In contrast to[15], we do not require any global information of power at each election round. Moreover, our examination of OELP isn't just asymptotic, i.e. the examination applies similarly well to small-sized network systems. Finally, OELP is scalable as it does not require any information of the exact position of every sensor node in the field. On the other hand, what we determined is a static RRT agreeing to the initial power of the sensor nodes which will remain same amid the entirety process. While power disseminates round after round, a potential enhancement is to dynamically process the correct RRT as per the current remained power of sensor nodes round after round, which will be additionally researched in the future work.

\section{References}

[1] Sun Y, Gurewitz O, Johnson DB., 2008 Nov 5. RI-MAC. a receiver-initiated asynchronous duty cycle $\mathrm{MAC}$ protocol for dynamic traffic loads in wireless sensor networks. InProceedings of the 6th ACM conference on Embedded network sensor systems, pp.1-14.

[2] Kim, J., Lin, X., Shroff, N.B. and Sinha, P., 2009. Minimizing delay and maximizing lifetime for wireless sensor networks with anycast. IEEE/ACM Transactions on networking, 18(2), pp.515528.

[3] Heinzelman, W.R., Chandrakasan, A. and Balakrishnan, H., 2000 , January. Energy-efficient communication protocol for wireless microsensor networks. In Proceedings of the 33rd annual Hawai international conference on system sciences,IEEE, pp.3005-3014.
[4] Smaragdakis, G., Matta, I. and Bestavros, A., 2004. SEP: A stable election protocol for clustered heterogeneous wireless sensor networks. Boston University Computer Science Department

[5] Li, D. and Wei, S., 2017, May. Improvement of the SEP protocol based on community structure of node degree. In AIP Conference Proceedings, vol. 1839, no. 1, p. 020218. AIP Publishing LLC.

[6] Wu Yucheng, Xie L., 2014. Distributed Energy Efficient Unequal Multi-Hop Clustering Algorithm for Wireless Sensor Networks.JOURNAL OF JIANGSU UNIVERSITY (Natural Science Edition), 35(2), pp. 196-200.

[7] ZHOU, F., LI, L.Y., DAI, J.J. and PENG, Y., 2013. A Clustering Multi-Hop Routing ProtocolEB-LEACH Based on Energy Balance.Journal of North University of China (Natural Science Edition), 34(4), pp.413-418.

[8] W. R. Heinzelman, "An application-specific protocol architecture for wireless microsensor networks," IEEE Transactions on Wireless Communications, vol. 1, no. 4, pp. 660-670, October 2002.

[9] W. R. Heinzelman, "Application-Specific Protocol Architectures for Wireless Networks," PhD Thesis, Massachusetts Institute of Technology, 2000.

[10] Rostami, A.S., Badkoobe, M., Mohanna, F., Hosseinabadi, A.A.R. and Sangaiah, A.K., 2018. Survey on clustering in heterogeneous and homogeneous wireless sensor networks. The Journal of Supercomputing, 74(1), pp.277-323.

[11] Dong, M., Ota, K. and Liu, A., 2016. RMER: Reliable and energyefficient data collection for large-scale wireless sensor networks. IEEE Internet of Things Journal, 3(4), pp.511-519.

[12] Chandanse, A., Bharane, P., Anchan, S. and Patil, H., 2019. Performance Analysis of Leach Protocol in Wireless Sensor Network.2nd International Conference on Advances in Science \& Technology (ICAST).

[13] Akyildiz, I.F. and Vuran, M.C., 2010. Wireless sensor networks (Vol. 4). John Wiley \& Sons.

[14] Mao, Y., Li, X. and Yang, S.X., 2011. An effective data gathering scheme in heterogeneous energy wireless sensor networks. Intelligent Automation \& Soft Computing, 17(8), pp.1023-1034.

[15] W. R. Heinzelman, 2000. Application-Specific Protocol Architectures for Wireless Networks. Ph.D. thesis, Massachusetts Institute of Technology. 